\title{
Reverse-Link Capacity of Power-Controlled CDMA Systems With Beamforming
}

\author{
Jin Yu, Student Member, IEEE, Yu-Dong Yao, Senior Member, IEEE, and Jinyun Zhang
}

\begin{abstract}
In this paper, reverse-link capacity, in terms of user capacity and Erlang capacity, of a direct-sequence code-division multiple-access (DS-CDMA) system with the use of beamforming is investigated. Signal-to-interference ratio (SIR)-based power control is assumed and both transmit and receive beamforming are considered. Instead of using tedious iterative methods to evaluate user capacity, a simple closed-form capacity expression with respect to antenna gains, a target SIR, and the CDMA processing gain is derived. Numerical results indicate significant capacity improvement with beamforming. The impact of the estimation errors of arrival angles on the capacity is examined. The joint use of a RAKE receiver and beamforming is investigated and the capacity expression for CDMA systems with multiclass services is also derived.
\end{abstract}

Index Terms-Beamforming, code-division multiple access (CDMA), Erlang capacity, power control, RAKE receiver, user capacity.

\section{INTRODUCTION}

W ITH the advance of wireless communication technology, there is an explosive increase in the number of mobile users. Although second-generation $(2 \mathrm{G})$ wireless systems, such as the global system for mobile communications (GSM) and IS-95 are successful in many countries [1], they still cannot meet the requirement of high-speed data and user capacity in high-user-density areas. Higher system capacity, better quality of service (QoS), and flexible accommodations of various wide-band services (such as video and multimedia services) with different transmission rates are required in third-generation $(3 \mathrm{G})$ wireless communication systems [2]. Code-division multiple access (CDMA) has been chosen as the radio interface technology for 3G systems [3]. Unlike frequency-division multiple access (FDMA) and time-division multiple access (TDMA), which are primarily bandwidth or dimension limited in capacity, CDMA capacity is interference limited [4]. Thus, any reduction of the interference will directly lead to capacity increases. The emerging technologies, such as beamforming and multiuser detections, could lead to a significant reduction in the interference and result in many-fold capacity increases

Manuscript received December 7, 2002; revised September 10, 2003, January 23, 2004, March 26, 2004, and May 7, 2004.

J. Yu and Y.-D. Yao are with the Wireless Information Systems Engineering Laboratory (WISELAB), Department of Electrical and Computer Engineering, Stevens Institute of Technology, Hoboken, NJ 07030 USA (e-mail: jyu@stevens.edu; yyao@stevens.edu).

J. Zhang is with the Mitsubishi Electric Research Laboratories (MERL), Cambridge, MA 02139 USA (e-mail: jzhang@ @erl.com).

Digital Object Identifier 10.1109/TVT.2004.833618
[5]-[7]. Capacity estimation is an important element in the design of CDMA systems and in the performance evaluation of the new technologies.

Gilhousen et al. [4] estimated CDMA reverse-link user capacity, considering voice traffic only, where strength-based power control [8], [9] is assumed in the CDMA systems and total other-cell interference $I$ is modeled as Gaussian noise [4], [10]. $I$ increases with the number of active users per cell $N$, which results in a decrease of signal-to-interference ratio (SIR). The maximum $N$ can be found considering a target SIR $\gamma_{0}$. Using similar methods as [4], Kim and Sung estimated the reverse-link user capacity of SIR-based power-controlled CDMA systems in [11] and [12]. User capacity of multicode CDMA systems supporting voice and data traffic or heterogeneous constant-bit-rate traffic was analyzed in [13]. The effects of a RAKE receiver and antenna diversity on reverse-link user capacity are further investigated in [2]. The focus of this paper is to present the user-capacity gain of a SIR-based DS-CDMA system in a multicell and multipath fading environment obtained through the use of a RAKE receiver, transmit and receive beamforming and to derive a simple closed-form expression, which is related to the number of antennas and RAKE receiver fingers, a target signal-to-noise-plus-interference ratio (SNIR), and the CDMA processing gain, to estimate the reverse-link user capacity. The impact of the estimation errors of the arrival angles (used in beamforming) on the reverse-link user capacity is also examined. In CDMA performance evaluations, the system capacity has also been investigated in terms of Erlang capacity [14], [15], where the number of active users is considered to be a Poisson random variable. Note that the user capacity is used to measure CDMA systems with continuously active users, while Erlang capacity is used to measure CDMA systems with randomly active users [16]. Erlang capacity is defined as the average traffic load in terms of average number of users requesting service under a specified blocking probability requirement [14]. In this paper, user capacity is referred to as the number of users that a CDMA system could support at a desired SNIR without power constraints [6].

In this paper, the use of beamforming in CDMA systems is investigated and its impact on reverse-link user capacity is first analyzed. Both transmit and receive beamforming are considered in the system and the joint use of a RAKE receiver and beamforming is also examined. The capacity reduction due to the use of beamforming with inaccurate arrival angle estimation is examined. Traffic scenarios such as voice and multimedia services are considered in a multiclass CDMA system. A simple closed-form equation, which is related to the antenna array gain patterns, a target SNIR, and the CDMA processing 
gain is given to estimate the reverse-link user capacity. Furthermore, reverse-link Erlang capacity of a CDMA system with beamforming is studied.

This paper is organized as follows. System models, including beamforming and other-cell interference, are given in Section II. A closed-form expression to evaluate reverse-link user capacity of a CDMA cellular system with beamforming is derived in Section III. Section IV examines the impact of the estimation errors of the arrival angles on the capacity. Section $\mathrm{V}$ considers the use of a RAKE receiver and Section VI extends the results to multiclass CDMA systems. The Erlang capacity of a CDMA system with beamforming is analyzed in Section VII. Numerical results are given in Section VIII and, finally, the conclusion is drawn in Section IX.

\section{SYSTEM MODEL}

\section{A. Beamforming}

Beamforming has been widely used in wireless systems that employ a fixed set of antenna elements in an array. Considering receive beamforming in reverse-link transmissions, the signals from these antenna elements are combined to form a movable beam pattern that can be steered to a desired direction that tracks mobile stations (MSs) as they move. This allows the antenna system to focus radio frequency (RF) resources on a particular mobile station and to minimize the impact of interference [17], [18]. This is achieved using a beamformer by placing nulls at the directions of interference, while the antenna array gain in the direction of the desired transmitter is maintained to be constant. While few antenna elements could be installed at an MS, large antenna arrays can be implemented at a base station (BS). When beamforming is used at the MS, the transmit beam pattern can be adjusted to minimize the interference to unintended receivers (such as BSs in other cells). At a BS, receive beamforming for each desired user could be implemented independently without affecting the performance of other links [18].

A linear equally spaced (LES) array is considered here [Fig. 1(a)]. $\theta$ is the azimuthal angle and $\varphi$ is the elevation angle of a plane wave incident on the array. In this paper, the LES array is used for both transmit and receive beamforming. Considering a two-dimensional (2-D) multicell environment (in the horizontal plane) [17], we have $\varphi=90^{\circ}$. The distance $d$ between the elements of the LES array is assumed to be $0.5 \lambda$, where $\lambda$ is the carrier wavelength. In the LES array system, a combining network connects an array of low gain antenna elements and could generate an antenna pattern [17], [19]

$$
G(\theta, \phi)=\left|\frac{\sin (0.5 M \pi(\sin \phi-\sin \theta))}{M \sin (0.5 \pi(\sin \phi-\sin \theta))}\right|^{2}
$$

where $M$ is the number of antenna elements and $\phi$ is a variable. The beam could be steered to a desired direction by varying $\phi$. Considering $M=3$ and $\theta=30^{\circ}$, an LES array gain pattern is shown in Fig. 1(b). In the remainder of this paper, we will use the antenna pattern specified in (1) to evaluate the impact of beamforming on the CDMA reverse-link capacity.

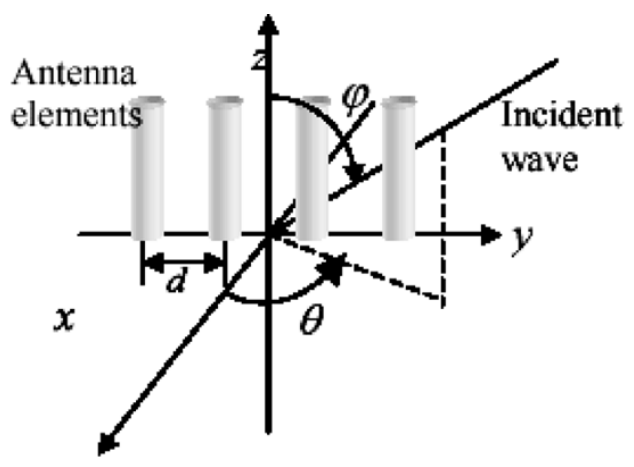

(a)

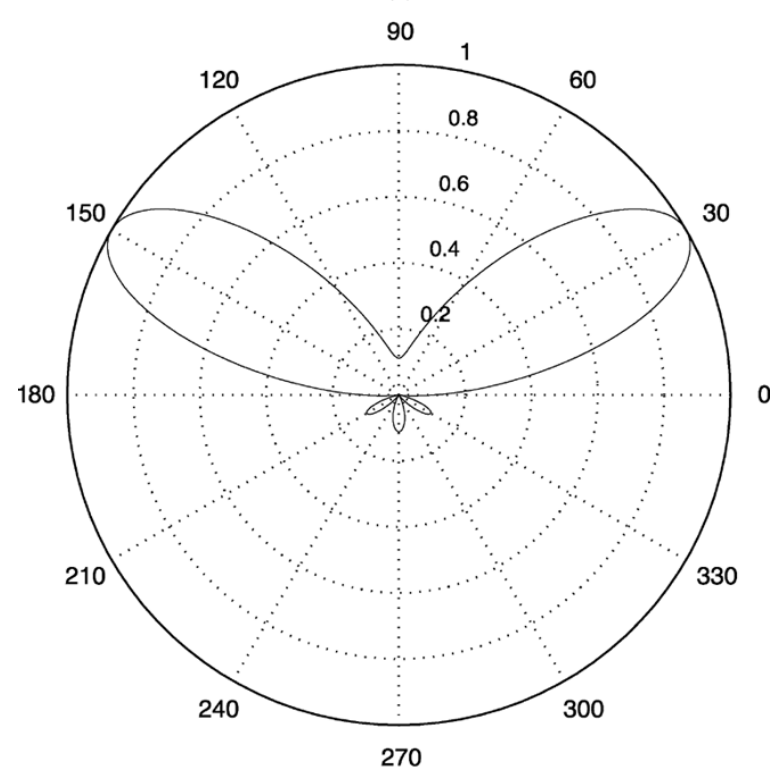

(b)

Fig. 1. (a) LES array and (b) LES array gain pattern with $M=3$ and $\theta=30^{\circ}$.

\section{B. Other-Cell Interference}

A cellular structure is shown in Fig. 2 with a reference cell $\left(\mathrm{BS}_{0}\right)$ and an interference cell (with BS BS $m$ ). In a CDMA cellular system, an MS is power controlled by a BS in its home cell to ensure that the received SNIR at the BS is no less than a target value, assuming that SIR-based power control is in use. Considering an MS MS $\mathrm{Mn}_{m, j}$ in the interference cell, let the received power at its BS $\left(\mathrm{BS}_{m}\right)$ be $S$. Considering the fact that fast fading does not affect the average power level [4], the received interference at the $\mathrm{BS}$ of the reference cell $\mathrm{BS}_{0}$ can be considered to be [11]

$$
I=S\left(\frac{r_{m}}{r_{0}}\right)^{\mu} 10^{\frac{\left(\xi_{0}-\xi_{m}\right)}{10}}
$$

where $r_{0}$ and $r_{m}$ are the distances from $\mathrm{MS}_{m, j}$ to $\mathrm{BS}_{0}$ and $\mathrm{BS}_{m}$, as shown in Fig. 2. $\mu$ is a path-loss exponent. $\xi_{0}$ and $\xi_{m}$ describe the shadowing processes in the cells of $\mathrm{BS}_{0}$ and $\mathrm{BS}_{m}$ and the shadowing processes are assumed to be mutually independent and follow a lognormal distribution with standard deviation $\sigma \mathrm{dB}$ and zero mean. Considering all interfering MSs, the total other-cell interference at $\mathrm{BS}_{0}$ is obtained by integrating the whole cellular coverage area except the reference cell [12]

$$
I=\iint S\left(\frac{r_{m}}{r_{0}}\right)^{\mu} 10^{\frac{\left(\xi_{0}-\xi_{m}\right)}{10}} \phi\left(\xi_{0}-\xi_{m}, \frac{r_{m}}{r_{0}}\right) \rho d A
$$




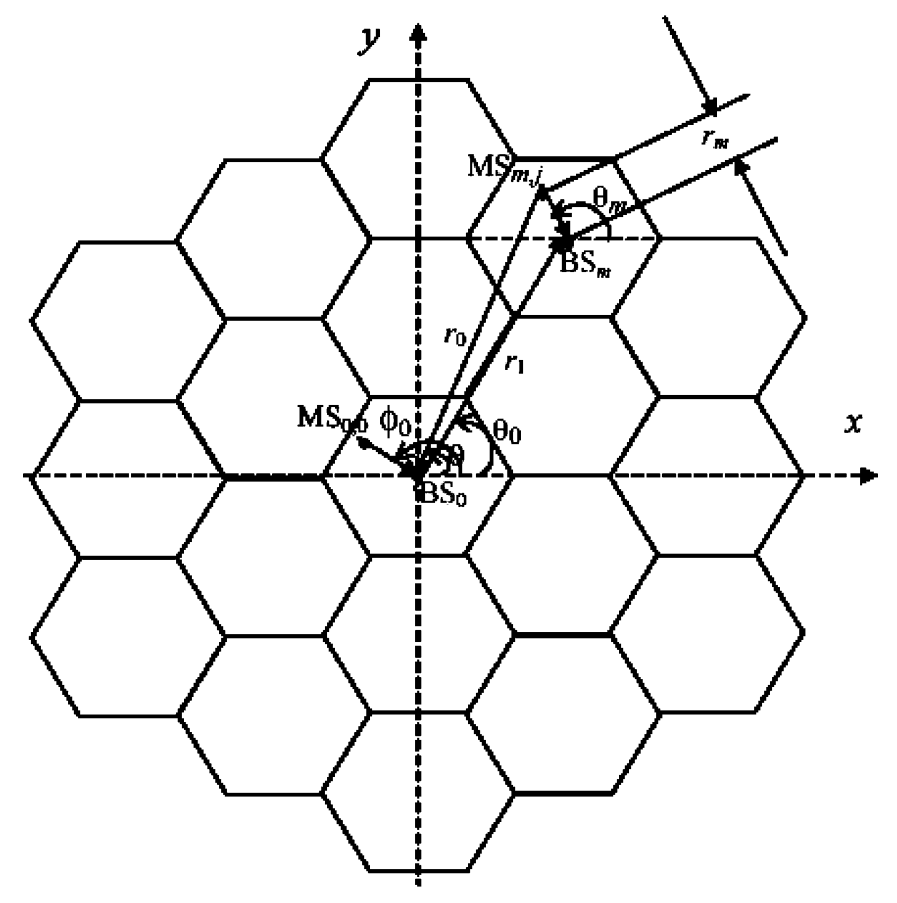

Fig. 2. Cellular structure and reverse-link geometry.

where

$$
\phi\left(\xi_{0}-\xi_{m}, \frac{r_{m}}{r_{0}}\right)=\left\{\begin{array}{lc}
1, & \text { if }\left(\frac{r_{m}}{r_{0}}\right)^{\mu} 10^{\frac{\left(\xi_{0}-\xi_{m}\right)}{10}} \leq 1 \\
0, & \text { otherwise }
\end{array}\right.
$$

and $\rho=2 N /(3 \sqrt{3})$ is the user density per unit area and there are $N$ MSs in each cell. This assumes that the users are uniformly distributed in a cell and that the radius of the hexagonal cell is normalized to unity. $\phi\left(\xi_{0}-\xi_{m}, r_{m} / r_{0}\right)$ is an indicator function to show the cell areas that are excluded in the calculation of $I$, since the MSs in these areas are not power controlled by $\mathrm{BS}_{m}$, but by $\mathrm{BS}_{0}$. In computing the above integral, we simply consider the hexagonal areas of each cell rather than the actual coverage area of the BSs [4], [11], [12].

\section{DERIVATION OF REVERSE-LINK USER CAPACITY}

When beamforming is applied at both transmit and receive sides, the total other-cell interference at $\mathrm{BS}_{0}$ can be expressed as

$$
\begin{array}{r}
I=\iint S\left(\frac{r_{m}}{r_{0}}\right)^{\mu} 10^{\frac{\left(\xi_{0}-\xi_{m}\right)}{10}} \phi\left(\xi_{0}-\xi_{m}, \frac{r_{m}}{r_{0}}\right) \\
\times \rho G_{t}\left(\theta, \theta_{m}\right) G_{r}\left(\theta, \phi_{0}\right) d A
\end{array}
$$

where

$$
\theta=\arctan \left(\frac{r_{1} \sin \theta_{0}+r_{m} \sin \theta_{m}}{r_{1} \cos \theta_{0}+r_{m} \cos \theta_{m}}\right)
$$

and $G_{t}\left(\theta, \theta_{m}\right)$ and $G_{r}\left(\theta, \phi_{0}\right)$ are transmit and receive beamforming gain patterns. $\theta_{m}$ and $\theta$ are the azimuth angle of $\mathrm{MS}_{m, j}$ to its home $\mathrm{BS} \mathrm{BS}_{m}$ and that to $\mathrm{BS}_{0}$, respectively. $r_{1}$ is the distance between $\mathrm{BS}_{m}$ and $\mathrm{BS}_{0} . \theta_{0}$ is the azimuth angle of $\mathrm{BS}_{m}$ to $\mathrm{BS}_{0} . \phi_{0}$ is the azimuth angle of $\mathrm{MS} \mathrm{MS}_{0,0}$ to $\mathrm{BS}_{0}$, as shown in Fig. 2, and is uniformly distributed from 0 to $2 \pi$. Fig. 3 indicates angle notations in transmit beamforming at $\mathrm{MS}_{m, j}$. When $M_{t}$ antenna elements are used with the beamforming pattern shown

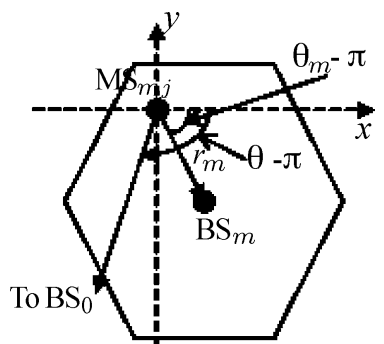

Fig. 3. Angle notations in transmit beamforming at $\mathrm{MS}_{m, j}$.

in (1), the transmit antenna gain in the direction from $\mathrm{MS}_{m, j}$ to $\mathrm{BS}_{0}$ is

$$
\begin{aligned}
G_{t}\left(\theta, \theta_{m}\right) & =\left|\frac{\sin \left(0.5 M_{t} \pi\left(\sin (\theta-\pi)-\sin \left(\theta_{m}-\pi\right)\right)\right)}{M_{t} \sin \left(0.5 \pi\left(\sin (\theta-\pi)-\sin \left(\theta_{m}-\pi\right)\right)\right)}\right|^{2} \\
& =\left|\frac{\sin \left(0.5 M_{t} \pi\left(\sin \theta-\sin \theta_{m}\right)\right)}{M_{t} \sin \left(0.5 \pi\left(\sin \theta-\sin \theta_{m}\right)\right)}\right|^{2}
\end{aligned}
$$

Similarly, when receive beamforming is applied with $M_{r}$ antenna elements at $\mathrm{BS}_{0}$ for receiving signals from $\mathrm{MS}_{0,0}$, the receive antenna gain in the direction from $\mathrm{MS}_{m, j}$ to $\mathrm{BS}_{0}$ is

$$
G_{r}\left(\theta, \phi_{0}\right)=\left|\frac{\sin \left(0.5 M_{r} \pi\left(\sin \theta-\sin \phi_{0}\right)\right)}{M_{r} \sin \left(0.5 \pi\left(\sin \theta-\sin \phi_{0}\right)\right)}\right|^{2} .
$$

The expected value of the total other-cell interference is

$$
\begin{aligned}
\mathrm{E}[I]=\mathrm{E}\left[\iint\right. & S\left(\frac{r_{m}}{r_{0}}\right)^{\mu} 10^{\frac{\left(\xi_{0}-\xi_{m}\right)}{10}} \\
& \left.\times \phi\left(\xi_{0}-\xi_{m}, \frac{r_{m}}{r_{0}}\right) \rho G_{t}\left(\theta, \theta_{m}\right) G_{r}\left(\theta, \phi_{0}\right) d A\right]
\end{aligned}
$$

which can be rewritten as

$$
\mathrm{E}[I]=\mathrm{E}[S] F(\mu, \sigma) N
$$

with

$$
\begin{aligned}
F(\mu, \sigma)= & \frac{2}{3 \sqrt{3}} \exp \left\{\left(\frac{\sigma \ln (10)}{10}\right)^{2}\right\} \\
& \times \iint\left(\frac{r_{m}}{r_{0}}\right)^{\mu} G_{t}\left(\theta, \theta_{m}\right) \\
& \times Q\left(\frac{10 \mu}{\sqrt{2 \sigma^{2}}} \log _{10}\left(\frac{r_{0}}{r_{m}}-\sqrt{2 \sigma^{2}} \frac{\ln (10)}{10}\right)\right) \\
& \times \mathrm{E}_{\phi_{0}}\left[G_{r}\left(\theta, \phi_{0}\right)\right] d A
\end{aligned}
$$

where

$$
\mathrm{E}_{\phi_{0}}\left[G_{r}\left(\theta, \phi_{0}\right)\right]=\frac{1}{2 \pi} \int_{0}^{2 \pi} G_{r}\left(\theta, \phi_{0}\right) d \phi_{0}
$$

and

$$
Q(x)=\frac{1}{\sqrt{2 \pi}} \int_{x}^{\infty} \exp \left(-\frac{t^{2}}{2}\right) d t .
$$


Similarly, the variance of $I$ is expressed as

$$
\begin{aligned}
\operatorname{Var}[I]=\operatorname{Var}[ & \iint S\left(\frac{r_{m}}{r_{0}}\right)^{\mu} 10^{\frac{\left(\xi_{0}-\xi_{m}\right)}{10}} \\
& \left.\times \phi\left(\xi_{0}-\xi_{m}, \frac{r_{m}}{r_{0}}\right) \rho G_{t}\left(\theta, \theta_{m}\right) G_{r}\left(\theta, \phi_{0}\right) d A\right]
\end{aligned}
$$

which, following [4], is rewritten as

$$
\operatorname{Var}[I]=\left\{U(\mu, \sigma) \mathrm{E}\left[S^{2}\right]-V(\mu, \sigma) \mathrm{E}^{2}[S]\right\} N
$$

where

$$
\begin{aligned}
U(\mu, \sigma)= & \frac{2}{3 \sqrt{3}} \exp \left\{\left(\frac{\sigma \ln (10)}{5}\right)^{2}\right\} \\
& \times \iint\left(\frac{r_{m}}{r_{0}}\right)^{2 \mu} G_{t}^{2}\left(\theta, \theta_{m}\right) \\
& \times Q\left(\frac{10 \mu}{\sqrt{2 \sigma^{2}}} \log _{10}\left(\frac{r_{0}}{r_{m}}-\sqrt{2 \sigma^{2}} \frac{\ln (10)}{5}\right)\right) \\
& \times \mathrm{E}_{\phi_{0}}\left[G_{r}^{2}\left(\theta, \phi_{0}\right)\right] d A \\
V(\mu, \sigma)= & 2 \sqrt{3}^{2} \exp \left\{2\left(\frac{\sigma \ln (10)}{10}\right)^{2}\right\} \\
& \times \iint\left(\frac{r_{m}}{r_{0}}\right)^{2 \mu} G_{t}^{2}\left(\theta, \theta_{m}\right) \\
& \times Q^{2}\left(\frac{10 \mu}{\sqrt{2 \sigma^{2}}} \log _{10}\left(\frac{r_{0}}{r_{m}}-\sqrt{2 \sigma^{2}} \frac{\ln (10)}{10}\right)\right) \\
& \times \mathrm{E}_{\phi_{0}}^{2}\left[G_{r}\left(\theta, \phi_{0}\right)\right] d A
\end{aligned}
$$

and

$$
\mathrm{E}_{\phi_{0}}\left[G_{r}^{2}\left(\theta, \phi_{0}\right)\right]=\frac{1}{2 \pi} \int_{0}^{2 \pi} G_{r}^{2}\left(\theta, \phi_{0}\right) d \phi_{0} .
$$

The value $F(\mu, \sigma), U(\mu, \sigma)$, and $V(\mu, \sigma)$ can be obtained numerically. For example, when only the first- and second-tier cells are considered, we find, for $\mu=4, \sigma=8 \mathrm{~dB}, M_{r}=3$, and $M_{t}=1, F(4,8)=0.2676, U(4,8)=0.1072$, and $V(4,8)=$ 0.0197 .

In CDMA systems, the received SNIR $E_{b} / I_{0}$ should be no less than a target value $\gamma_{0}$ in order to maintain a required transmission quality. Following [2] and [11] and considering transmit and receive beamforming, we have

$$
\begin{aligned}
\frac{E_{b}}{I_{0}} & \approx \frac{G S G_{t}\left(\phi_{0}, \phi_{0}\right) G_{r}\left(\phi_{0}, \phi_{0}\right)}{\frac{2}{3}\left(\sum_{m=1}^{N-1} S \mathrm{E}_{\phi_{0}, \phi_{m}}\left[G_{t}\left(\phi_{m}, \phi_{m}\right) G_{r}\left(\phi_{m}, \phi_{0}\right)\right]+I\right)+\eta_{0} W} \\
& =\frac{G S}{\frac{2}{3}\left(\sum_{m=1}^{N-1} S \mathrm{E}_{\phi_{0}, \phi_{m}}\left[G_{r}\left(\phi_{m}, \phi_{0}\right)\right]+I\right)+\eta_{0} W} \geq \gamma_{0}
\end{aligned}
$$

where $G$ is the CDMA processing gain, $\eta_{0}$ is the single-sided white noise power spectrum density, and $W$ is the spreading bandwidth. The factor $2 / 3$ in the denominator is due to the assumption of a square chip pulse. The denominator in (7) includes other-cell interference as well as own-cell interference due to other MSs in the reference cell. Note that the transmit antenna gains of MSs at the reference cell in (7) are all set to

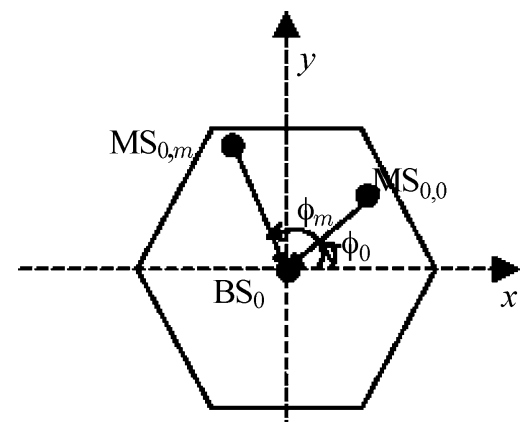

Fig. 4. Angle notations of receive beamforming at $\mathrm{BS}_{0}$.

unity since their transmit beams are steered toward $\mathrm{BS} \mathrm{BS}_{0}$. $\phi_{m}$ is the azimuth angle of an interfering $\mathrm{MS} \mathrm{MS}_{0, m}$ to $\mathrm{BS}_{0}$. Fig. 4 illustrates angle notations of receive beamforming at $\mathrm{BS}_{0}$. $G_{r}\left(\phi_{m}, \phi_{0}\right)$ is the receive beamforming gain of $\mathrm{MS}_{0,0}$ to the direction of $\mathrm{MS}_{0, m} . \phi_{m}$ and $\phi_{0}$ are uniformly distributed in $[0,2 \pi)$. Let $\phi=\sin \phi_{m}-\sin \phi_{0}$; the probability density function (pdf) of $\phi$ is found to be

$$
f(\phi)=\left\{\begin{array}{cl}
\frac{1}{\pi^{2}} \int_{-1}^{\phi+1} \frac{1}{\sqrt{\left(1-\tau^{2}\right)\left(1-(\phi-\tau)^{2}\right)}} d \tau, & -2<\phi<0 \\
\frac{1}{\pi^{2}} \int_{\phi-1}^{1} \frac{1}{\sqrt{\left(1-\tau^{2}\right)\left(1-(\phi-\tau)^{2}\right)}} d \tau, & 0 \leq \phi<2 \\
0, & \text { otherwise }
\end{array}\right.
$$

We further have

$$
\mathrm{G}_{\mathrm{r}}=\mathrm{E}_{\phi_{0}, \phi_{m}}\left[G_{r}\left(\phi_{m}, \phi_{0}\right)\right]=\int_{-2}^{2} \frac{\sin ^{2}\left(0.5 M_{r} \pi \phi\right)}{M_{r}^{2} \sin ^{2}(0.5 \pi \phi)} f(\phi) d \phi .
$$

Let $S$ be the minimum power level satisfying (7). The received power $S$ could be expressed in terms of $I$ as

$$
S=\frac{I+1.5 \eta_{0} W}{C}
$$

where

$$
C=\frac{1.5 G}{\gamma_{0}}-\mathrm{G}_{\mathrm{r}}(N-1)
$$

Now, the user capacity $N$ can be found via an iterative method [2], [11], in which there are two concatenated iteration loops. In the inner loop, for a given $N$ value, determine $\mathrm{E}[I]$ and $\operatorname{Var}[I]$ using the following steps.

Step 1) Set E $[I]$ and $\operatorname{Var}[I]$ as zeros.

Step 2) Calculate $\mathrm{E}[S]$ and $\mathrm{E}\left[S^{2}\right]$ from (8).

Step 3) Calculate E[I] and $\operatorname{Var}[I]$ from (5) and (6).

Step 4) Repeat Steps 2) and 3) until the differences between old and new values of $\mathrm{E}[I]$ and $\operatorname{Var}[I]$ are less than $1 \%[2]$.

Using the $\mathrm{E}[I]$ and $\operatorname{Var}[I]$ obtained above and a specified maximum transmission power limit, calculate an outage probability (the transmission power exceeds the power constraint) [2]. If the outage probability does not exceeds a required level, the outer loop increases $N$ by 1 and enters the interloop. The iteration loops stop when the calculated outage probability exceeds the required level. We then obtain the user capacity as $N-1$. 
The iterative method to determine user capacity as described above is computationally complicated due to multiple loops of numerical integrations. In this paper, we derive a formula to directly estimate user capacity, which is related to all the relevant factors, such as $\gamma_{0}$, beamforming gains, and CDMA processing gains. Solving (5), (6), and (8), we get

$$
\begin{aligned}
\mathrm{E}[I] & =\frac{1.5 N F(\mu, \sigma)}{C-N F(\mu, \sigma)} \eta_{0} W \\
\operatorname{Var}[I] & =\frac{(U(\mu, \sigma)-V(\mu, \sigma))}{\frac{C^{2}}{N}-U(\mu, \sigma)}\left(\mathrm{E}[I]+1.5 \eta_{0} W\right)^{2} .
\end{aligned}
$$

It is obvious that $\mathrm{E}[I]$ and $\operatorname{Var}[I]$ are greater than 0 because the noise or interference power is always positive. Thus, a valid $N$ has to ensure that (9) and (10) are greater than 0. As shown in Appendix A, we are able to determine user capacity $N$ as

$$
N=\left\lfloor\frac{\mathrm{G}_{\mathrm{r}}+\frac{1.5 G}{\gamma_{0}}}{\mathrm{G}_{\mathrm{r}}+F(\mu, \sigma)}\right\rfloor
$$

where $\lfloor x\rfloor$ indicates maximum integer no greater than $x$. In using the iterative method to determine the user capacity, we found that when the number of users is above the user capacity, power control will not work, since every MS tries to satisfy the target SNIR $\gamma_{0}$ by increasing its transmit power until reaching its maximum transmit power allowed. This leads to an increase of interference to other users. The results from the closed-form expression (11) will be compared in Section VIII with those results obtained from the iterative method.

\section{IMPACT OF DoA Mismatch}

In this section, the impact of direction of arrival (DoA) mismatch on the capacity is analyzed. The arrival angle, $\theta$, can be characterized as a random variable with a uniform distribution or a normal distribution [20],

$$
f(\theta)= \begin{cases}\frac{1}{2 \sqrt{3} \triangle},-\sqrt{3} \triangle \leq\left(\theta-\theta_{s}\right) \leq \sqrt{3} \triangle, & \text { unif. } \\ \frac{1}{\sqrt{2 \pi} \triangle} \exp \left\{-\frac{\left(\theta-\theta_{s}\right)^{2}}{2 \triangle^{2}}\right\}, & \text { norm. }\end{cases}
$$

where $\theta_{s}$ is the exact arrival angle and $\triangle^{2}$ represents the variance of the angle estimation errors. Assuming perfect transmit power control, the transmit power of the $m$ th user in other cell is

$$
P_{T}=\frac{S r_{m}^{\mu} 10^{\frac{\xi_{m}}{10}}}{\left(G_{t}\left(\theta_{m}^{s}, \theta_{m}\right) G_{r}\left(\phi_{0}^{s}, \phi_{0}\right)\right)}
$$

where $\theta_{m}$ is the estimated departure angle of the $m$ th user in other cell and $\phi_{0}$ represents the estimated arrival angle of the desired user. Therefore, the total interference $I$ is

$$
\begin{aligned}
I=\iint S\left(\frac{r_{m}}{r_{0}}\right)^{\mu} 10^{\frac{\left(\xi_{0}-\xi_{m}\right)}{10}} & \phi\left(\xi_{0}-\xi_{m}, \frac{r_{m}}{r_{0}}\right) \\
& \times \rho \frac{G_{t}\left(\theta, \theta_{m}\right) G_{r}\left(\theta, \phi_{0}\right)}{G_{t}\left(\theta_{m}^{s}, \theta_{m}\right) G_{r}\left(\phi_{0}^{s}, \phi_{0}\right)} d A .
\end{aligned}
$$

Thus, we have $\mathrm{E}[I]=\mathrm{E}[S] N F(\mu, \sigma)$ with

$$
\begin{aligned}
F(\mu, \sigma)= & \frac{2}{3 \sqrt{3}} \exp \left\{\left(\frac{\sigma \ln (10)}{10}\right)^{2}\right\} \\
& \times \iint\left(\frac{r_{m}}{r_{0}}\right)^{\mu} \mathrm{E}_{\theta_{m}}\left[\frac{G_{t}\left(\theta, \theta_{m}\right)}{G_{t}\left(\theta_{m}^{s}, \theta_{m}\right)}\right] \\
& \times Q\left(\frac{10 \mu}{\sqrt{2 \sigma^{2}}} \log _{10}\left(\frac{r_{0}}{r_{m}}-\sqrt{2 \sigma^{2}} \frac{\ln (10)}{10}\right)\right) \\
& \times \mathrm{E}_{\phi_{0}, \phi_{0}^{s}}\left[\frac{G_{r}\left(\theta, \phi_{0}\right)}{G_{r}\left(\phi_{0}^{s}, \phi_{0}\right)}\right] d A
\end{aligned}
$$

where

$$
\mathrm{E}_{\phi_{0}, \phi_{0}^{s}}\left[\frac{G_{r}\left(\theta, \phi_{0}\right)}{G_{r}\left(\phi_{0}^{s}, \phi_{0}\right)}\right]=\frac{1}{2 \pi} \int_{0}^{2 \pi} \int f\left(\phi_{0}\right) \frac{G_{r}\left(\theta, \phi_{0}\right)}{G_{r}\left(\phi_{0}^{s}, \phi_{0}\right)} d \phi_{0} d \phi_{0}^{s}
$$

and

$$
\mathrm{E}_{\theta_{m}}\left[\frac{G_{t}\left(\theta, \theta_{m}\right)}{G_{t}\left(\theta_{m}^{s}, \theta_{m}\right)}\right]=\int f\left(\theta_{m}\right) \frac{G_{t}\left(\theta, \theta_{m}\right)}{G_{t}\left(\theta_{m}^{s}, \theta_{m}\right)} d \theta_{m}
$$

where $\phi_{0}^{s}$ is the exact arrival angle of the desired user, which is also the mean value of $\phi_{0}$, due to the uniform or normal distribution of the DoA estimation errors. The SNIR with DoA mismatch assuming that perfect power control can be derived as

$$
\frac{E_{b}}{I_{0}}=\frac{G S}{\frac{2}{3}\left((N-1) S \hat{\mathrm{G}}_{\mathrm{r}}+I\right)+\eta_{0} W} \geq \gamma_{0}
$$

where

$$
\begin{aligned}
\hat{\mathrm{G}}_{\mathrm{r}}= & \mathrm{E}_{\phi_{0}, \phi_{m}^{s}, \phi_{0}^{s}}\left[\frac{G_{r}\left(\phi_{m}^{s}, \phi_{0}\right)}{G_{r}\left(\phi_{0}^{s}, \phi_{0}\right)}\right] \\
= & \frac{1}{4 \pi^{2}} \int_{0}^{2 \pi} \int_{0}^{2 \pi} \int \frac{f\left(\phi_{0}\right)}{G_{r}\left(\phi_{0}^{s}, \phi_{0}\right)} \\
& \times\left|\frac{\sin \left(0.5 M \pi\left(\sin \phi_{m}^{s}-\sin \phi_{0}\right)\right)}{M \sin \left(0.5 \pi\left(\sin \phi_{m}^{s}-\sin \phi_{0}\right)\right)}\right|^{2} d \phi_{0} d \phi_{m}^{s} d \phi_{0}^{s}
\end{aligned}
$$

where $\phi_{m}^{s}$ represents the exact arrival angles of the $m$ th user of the reference cell. Similarly, since all the users are uniformly distributed in the cell, the expected gain $\hat{\mathrm{G}}_{\mathrm{r}}$ have to be averaged throughout the whole cell area. Considering the conditions $\mathrm{E}[I]>0$ and $\operatorname{Var}[I]>0$, the reverse-link capacity with DoA estimation errors can be found to be

$$
N=\left\lfloor\frac{\hat{\mathrm{G}}_{\mathrm{r}}+\frac{1.5 G}{\gamma_{\mathrm{o}}}}{\hat{\mathrm{G}_{\mathrm{r}}}+F(\mu, \sigma)}\right\rfloor .
$$

Note that the issue of angular spreads is not addressed here. For rural environments, angular spreads between $1-5^{\circ}$ have been observed [21]. For urban and hilly terrain environments, considerably larger angular spreads, as long as $20^{\circ}$, have been found [22]. The impact of the angular spread on CDMA capacity will be investigated in future studies. 


\section{CONSIDERATION OF THE RAKE RECEIVER}

Multipath propagation in the radio channel leads to deep fading of the received signal. If the paths are independent and resolvable in time domain, i.e., the delay between different paths is greater than the chip duration $T_{c}$, a RAKE receiver can be used to combine the paths to achieve diversity gains [15]. The multipath fading can be characterized by a power delay profile (PDP), which is uniformly or exponentially distributed [2]. For a uniform PDP, we have

$$
\mathrm{E}\left[a_{l}\right]=\frac{1}{L}, \quad l=0,1, \cdots, L-1
$$

and for an exponential PDP we have

$$
\mathrm{E}\left[a_{l}\right]=(1-\exp (-\varepsilon)) \exp (-\varepsilon l), \quad l \geq 0
$$

where $a_{l}$ is the square path gain of the $l$ th path and follows an exponential distribution (its envelop is Rayleigh distributed), $L$ is the total number of paths in a uniform profile, and $\epsilon$ is a decay factor for an exponential profile. Assuming that the RAKE receiver at the BS has $R$ fingers, the received power at the output is the combination of the $R$ paths $X=a_{0}+a_{1}+\cdots+a_{R-1}$. Following [2], (5), (6), and (7) are modified as

$$
\begin{aligned}
\mathrm{E}[I] & =\mathrm{E}[S] F(\mu, \sigma) N \mathrm{E}\left[\frac{1}{X}\right] \\
\operatorname{Var}[I] & =\left(U(\mu, \sigma) \mathrm{E}\left[S^{2}\right]-V(\mu, \sigma) \mathrm{E}^{2}[S]\right) N \mathrm{E}^{2}\left[\frac{1}{X}\right]
\end{aligned}
$$

and

$$
\frac{E_{b}}{I_{0}}=\frac{G S}{\frac{2}{3}\left(S \mathrm{G}_{\mathrm{r}} N(1+D)+I\right)+\eta_{0} W} \geq \gamma_{0}
$$

where

$$
D=\left\{\begin{array}{lc}
\frac{\mathrm{E}\left[\frac{1}{X}\right](L-R)}{R}, & \text { for uniform PDP } \\
\mathrm{E}\left[\frac{1}{X}\right] \exp (-\epsilon R), & \text { for exponential PDP. }
\end{array}\right.
$$

We can get the expression of $S$ in terms of $I$ when SNIR satisfies the minimum requirement $\gamma_{0}$

$$
S=\frac{I+1.5 \eta_{0} W}{\frac{1.5 G}{\gamma_{0}}-\mathrm{G}_{\mathrm{r}} N(1+D)}
$$

Considering $\mathrm{E}[I]>0$ and $\operatorname{Var}(I)>0$ and using (14)-(16), the user capacity with a joint use of a RAKE receiver and beamforming can be derived as

$$
N=\left\lfloor\frac{\frac{1.5 G}{\gamma_{0}}}{\mathrm{G}_{\mathrm{r}}(1+D)+\mathrm{E}\left[\frac{1}{X}\right] F(\mu, \sigma)}\right\rfloor .
$$

\section{ANALYSIS OF MulTiClass CDMA}

In Section III, we derived the user capacity in a flat-fading environment. Multipath propagation (resolvable multipaths) and the use of a RAKE receiver are considered in Section IV. In this section, we extend the results to a CDMA system with multiclass operation. Multiclass CDMA is a method to support users requiring varying date rates for different applications. In the multiclass CDMA system, a single code can be assigned to each service (such as audio, data, and video services) with different processing gains. This differs from multicode CDMA [23], which can also be used to realize the multiclass CDMA. Heterogeneous constant-bit-rate (CBR) traffic, such as audio, data, and video services, are considered in this paper, which are assigned different spreading codes with processing gains $G_{a}$, $G_{d}$, and $G_{v}$, respectively. For example, we could assume that spreading gains for audio, data, and video traffic are 128, 64, and 32 , respectively. In this way, the services with different rates are accommodated with spreading sequences with various lengths or processing gains. If the processing gains are normalized by $G=G_{a}=128$, we obtain the normalized processing gains for audio, data, and video services $g_{a}=1, g_{d}=1 / 2$, and $g_{v}=1 / 4$. The total other-cell interference comes from all the traffic types is [11] $I=I_{a}+I_{d}+I_{v}$. If the traffics are assumed to be independent of each other, we could get

$$
\begin{aligned}
\mathrm{E}[I] & =\mathrm{E}\left[I_{a}\right]+\mathrm{E}\left[I_{d}\right]+\mathrm{E}\left[I_{v}\right] \\
\operatorname{Var}[I] & =\operatorname{Var}\left[I_{a}\right]+\operatorname{Var}\left[I_{d}\right]+\operatorname{Var}\left[I_{v}\right] .
\end{aligned}
$$

Note that three types of spreading sequences with different processing gains, $G g_{a}, G g_{d}$, and $G g_{v}$, are used to meet the requirement for different rates. Furthermore, in order to meet SNIR requirements $\left(\gamma_{a}, \gamma_{d}, \gamma_{v}\right)$ for different services, the corresponding transmission power is controlled to obtain the required received power for each service $\left(P_{a}, P_{d}\right.$, and $\left.P_{v}\right)$. And different traffic types have to satisfy the different SNIR requirements

$$
\begin{aligned}
\left(\frac{E_{b}}{I_{0}}\right)_{a} & =\frac{G_{a} P_{a}}{\frac{2}{3}\left(\left(N_{a}-1\right) P_{a} \mathrm{G}_{\mathrm{r}}+N_{d} P_{d} \mathrm{G}_{\mathrm{r}}+N_{v} P_{v} \mathrm{G}_{\mathrm{r}}+I\right)+\eta_{0} W} \\
\left(\frac{E_{b}}{I_{0}}\right)_{d} & =\frac{G_{d} P_{d}}{\frac{2}{3}\left(N_{a} P_{a} \mathrm{G}_{\mathrm{r}}+\left(N_{d}-1\right) P_{d} \mathrm{G}_{\mathrm{r}}+N_{v} P_{v} \mathrm{G}_{\mathrm{r}}+I\right)+\eta_{0} W} \\
& \geq \gamma_{d} \\
\left(\frac{E_{b}}{I_{0}}\right)_{v} & =\frac{G_{v} P_{v}}{\frac{2}{3}\left(N_{a} P_{a} \mathrm{G}_{\mathrm{r}}+N_{d} P_{d} \mathrm{G}_{\mathrm{r}}+\left(N_{v}-1\right) P_{v} \mathrm{G}_{\mathrm{r}}+I\right)+\eta_{0} W} \\
& \geq \gamma_{v}
\end{aligned}
$$

where subscripts $a, d$, and $v$ represent audio, data, and video traffic types, respectively. $N_{i}$ and $\gamma_{i}(i=a, d$, or $v)$ represent the corresponding user capacity and target SNIR, respectively. Let $S_{a}, S_{v}$, and $S_{d}$ be normalized received power $S_{a}=P_{a} g_{a}$, $S_{d}=P_{d} g_{d}, S_{v}=P_{v} g_{v}$. We rewrite the above equation as

$$
\begin{aligned}
& \frac{G S_{a}}{\frac{2}{3}\left(\frac{\left(N_{a}-1\right) S_{a} \mathrm{G}_{\mathrm{r}}}{g_{a}}+\frac{N_{d} S_{d} \mathrm{G}_{\mathrm{r}}}{g_{d}}+\frac{N_{v} S_{v} \mathrm{G}_{\mathrm{r}}}{g_{v}}+I\right)+\eta_{0} W} \geq \gamma_{a} \\
& \frac{G S_{d}}{\frac{2}{3}\left(\frac{N_{a} S_{a} \mathrm{G}_{\mathrm{r}}}{g_{a}}+\frac{\left(N_{d}-1\right) S_{d} \mathrm{G}_{\mathrm{r}}}{g_{d}}+\frac{N_{v} S_{v} \mathrm{G}_{\mathrm{r}}}{g_{v}}+I\right)+\eta_{0} W} \geq \gamma_{d} \\
& \frac{G S_{v}}{\frac{2}{3}\left(\frac{N_{a} S_{a} \mathrm{G}_{\mathrm{r}}}{g_{a}}+\frac{N_{d} S_{d} \mathrm{G}_{\mathrm{r}}}{g_{d}}+\frac{\left(N_{v}-1\right) S_{v} \mathrm{G}_{\mathrm{r}}}{g_{v}}+I\right)+\eta_{0} W} \geq \gamma_{v} .
\end{aligned}
$$

We could get the minimum required received power (normalized) $S_{a}, S_{d}$, and $S_{v}$ when we set the received $E_{b} / I_{0}$ of different traffic equal to their target values. Following [11] and [24] and 
solving (20), $S_{a}, S_{d}$, and $S_{v}$ could be expressed in terms of the total other-cell interference $I$ as

$$
\begin{aligned}
S_{a} & =\frac{\left(I+1.5 \eta_{0} W\right)}{C} \\
S_{d} & =\beta_{d} S_{a} \\
S_{v} & =\beta_{v} S_{a}
\end{aligned}
$$

where

$$
\begin{aligned}
& C=\frac{\mathrm{G}_{\mathrm{r}}}{g_{a}}+\frac{1.5 G}{\gamma_{a}}-\mathrm{G}_{\mathrm{r}}\left(\frac{N_{a}}{g_{a}}+\frac{N_{d} \beta_{d}}{g_{d}}+\frac{N_{v} \beta_{v}}{g_{v}}\right) \\
& \beta_{d}=\frac{\frac{1}{g_{a}}+\frac{1.5 G}{\mathrm{G}_{\mathrm{r}} \gamma_{a}}}{\frac{1}{g_{d}}+\frac{1.5 G}{\mathrm{G}_{\mathrm{r}} \gamma_{d}}} \\
& \text { and } \\
& \beta_{v}=\frac{\frac{1}{g_{a}}+\frac{1.5 G}{\mathrm{G}_{\mathrm{r}} \gamma_{a}}}{\frac{1}{g_{v}}+\frac{1.5 G}{\mathrm{G}_{\mathrm{r}} \gamma_{v} .}}
\end{aligned}
$$

Based on the derivations in Section III, the user capacity for a multiclass CDMA system in a flat fading channel is found as

$$
\frac{N_{a}}{g_{a}}+\frac{N_{d} \beta_{d}}{g_{d}}+\frac{N_{v} \beta_{v}}{g_{v}}=\left\lfloor\frac{\frac{\mathrm{G}_{\mathrm{r}}}{g_{a}}+\frac{1.5 G}{\gamma_{a}}}{\mathrm{G}_{\mathrm{r}}+F(\mu, \sigma)}\right\rfloor .
$$

When a multipath fading environment with resolvable paths is considered and a RAKE receiver is used in the multiclass CDMA system, the user capacity can be derived as

$$
\frac{N_{a}}{g_{a}}+\frac{N_{d} \beta_{d}}{g_{d}}+\frac{N_{v} \beta_{v}}{g_{v}}=\left\lfloor\frac{\frac{1.5 G}{\gamma_{a}}}{\mathrm{G}_{\mathrm{r}}(1+D)+\mathrm{E}\left[\frac{1}{X}\right] F(\mu, \sigma)}\right\rfloor_{(22)}
$$

where $\beta_{d}=\gamma_{d} / \gamma_{a}, \beta_{v}=\gamma_{v} / \gamma_{a}$, and $D$ and $\mathrm{E}[1 / X]$ are the same as given in Section V.

\section{ERLANG CAPACITY}

As discussed in Section I, the user capacity is used to measure CDMA systems with continuously active users and Erlang capacity is used to measure CDMA systems with randomly active users. We consider a system with a Poisson arrival and exponential service time in the Erlang capacity evaluation [24]. In Sections III-V, we investigated the user capacity over CDMA reverse links. In this section, we focus on the Erlang capacity and consider the use of beamforming.

Based on (7), total interference including own- and other-cell interference is $2\left[(N-1) \mathrm{G}_{\mathrm{r}} S+I\right] / 3$ and we have

$$
\frac{2}{3}\left[(N-1) \mathrm{G}_{\mathrm{r}} S+I\right] \leq \frac{G S}{\gamma_{0}}\left(1-\frac{\eta_{\mathrm{o}} W}{\frac{E_{b}}{\gamma_{0}}}\right)
$$

which can be rewritten as

$$
N \mathrm{G}_{\mathrm{r}}+\frac{I}{S} \leq \frac{1.5 G}{\gamma_{0}}(1-\eta)+\mathrm{G}_{\mathrm{r}}
$$

where

$$
\eta=\frac{\eta_{0} W}{\frac{E_{b}}{\gamma_{0}}}
$$

which is equal to the ratio of noise power to total noise-plus-interference power $\eta_{0} W / I_{0}$, when $E_{b} / I_{0}=\gamma_{0}$. The ratio of other-cell interference to own-cell interference plus signal power is

$$
f=\frac{\mathrm{E}[I]}{N \mathrm{E}[S] \mathrm{G}_{\mathrm{r}}}=\frac{F(\mu, \sigma)}{\mathrm{G}_{\mathrm{r}}}
$$

Let $Z=N+I / S \mathrm{G}_{\mathrm{r}}$ and $A=1+1.5 G(1-\eta) / \gamma_{0} \mathrm{G}_{\mathrm{r}}$. Following (21), we have $Z \leq A$. Now, we consider that a CDMA system has randomly active users with Poisson arrivals (mean call arrival rate $\lambda$ ) and exponential service time (mean call duration time $H$ ). A newly arrived user is blocked when $Z>A$ and, therefore, we have the blocking probability for a CDMA system

$$
P_{\text {blocking }}=\operatorname{P}_{\mathrm{r}}\{Z>A\} \text {. }
$$

According to [14], the random variable $Z$ can be approximated using the central limit theorem. The blocking probability is then

$$
P_{\text {blocking }}=Q\left[\frac{A-\mathrm{E}[Z]}{\sqrt{\operatorname{Var}(Z)}}\right] \text {. }
$$

The mean and variance of $Z$ can be obtained considering that, although the total number of other-cell users is generally much larger than that from a single cell, their average power is equivalent to that of $N f$ users [14]. The mean and variance of $Z$ are increased by a factor $(1+f)$, compared with own-cell interference

$$
\begin{aligned}
\mathrm{E}[Z] & =\mathrm{E}[N](1+f)=\lambda H(1+f) \\
\operatorname{Var}[Z] & =\operatorname{Var}[N](1+f)=\lambda H(1+f) .
\end{aligned}
$$

Substituting (25) and (26) into (24), we get

$$
\lambda H=\frac{A+\frac{B^{2}}{2}-B \sqrt{A+\frac{B^{2}}{4}}}{1+f}
$$

where

$$
B=Q^{-1}\left(P_{\text {blocking }}\right)
$$

Equation (27) gives the expression for reverse-link Erlang capacity of a CDMA system with beamforming, which relates to the call-blocking probability, target SNIR, CDMA processing gain, and expected antenna gain $\mathrm{G}_{\mathrm{r}}$.

\section{NUMERIC RESULTS}

Throughout this section, we assume propagation parameters $\mu=4$ and $\sigma=8 \mathrm{~dB}$. Table I lists multiclass CDMA system parameters. The basic data rate $R$ is assumed to be $32 \mathrm{~Kb} / \mathrm{s}$ and the spreading chip rate is $4.096 \mathrm{Mb} / \mathrm{s}$. The first traffic type is of the basic rate and its required SNIR target $\gamma_{a}$ is $5 \mathrm{~dB}$. The 
TABLE I

SYSTEM PARAMETERS

\begin{tabular}{c|c|c|c|c|c|c|c}
\hline$R$ & $W$ & $g_{a}$ & $g_{d}$ & $g_{v}$ & $\gamma_{a}$ & $\gamma_{d}$ & $\gamma_{v}$ \\
\hline $32 K b p s$ & $4.096 M b p s$ & 1 & 0.5 & 0.25 & $5 d B$ & $10 d B$ & $7 d B$ \\
\hline
\end{tabular}

TABLE II

COMPUTATIONAL PARAMETERS With $M_{t}=1$

\begin{tabular}{c|c|c|c|c|c}
\hline$\left(M_{t}, M_{r}\right)$ & $(1,1)$ & $(1,3)$ & $(1,5)$ & $(1,7)$ & $(1,9)$ \\
\hline$F(\mu, \sigma)$ & 0.6611 & 0.2676 & 0.1724 & 0.1287 & 0.1035 \\
\hline$U(\mu, \sigma)$ & 0.2252 & 0.1072 & 0.0691 & 0.0516 & 0.0414 \\
\hline$V(\mu, \sigma)$ & 0.0451 & 0.0197 & 0.0127 & 0.0094 & 0.0075 \\
\hline $\mathrm{G}_{\mathrm{r}}$ & 1 & 0.3855 & 0.2487 & 0.1863 & 0.1501 \\
\hline$\beta_{d}$ & 2.9111 & 3.0595 & 3.0950 & 3.1116 & 3.1213 \\
\hline$\beta_{v}$ & 1.5311 & 1.5635 & 1.5710 & 1.5744 & 1.5765 \\
\hline
\end{tabular}

TABLE III

COMPUTATIONAL PARAMETERS With $M_{t}=2$

\begin{tabular}{l|c|c|c|c|c}
\hline$\left(M_{t}, M_{r}\right)$ & $(2,1)$ & $(2,3)$ & $(2,5)$ & $(2,7)$ & $(2,9)$ \\
\hline$F(\mu, \sigma)$ & 0.3410 & 0.1458 & 0.0960 & 0.0726 & 0.0589 \\
\hline$U(\mu, \sigma)$ & 0.1329 & 0.0570 & 0.0379 & 0.0288 & 0.0234 \\
\hline$V(\mu, \sigma)$ & 0.0225 & 0.0100 & 0.0067 & 0.0051 & 0.0041 \\
\hline
\end{tabular}

second and third traffic types require higher data rates (64 and $128 \mathrm{~Kb} / \mathrm{s}$ ) and their SNIR targets are 10 and $7 \mathrm{~dB}$, respectively.

Table II considers a single transmit antenna element and, at the receive side, the number of antenna elements varies from $1,3,5,7$, to 9 . As $F(\mu, \sigma)$ is proportional to other-cell interference, we see that the value of $F(\mu, \sigma)$ decreases with an increase of the number of receive antenna elements, $M_{r}$. The received own-cell interference is proportional to the expected received antenna gain $\mathrm{G}_{\mathrm{r}}$, which decreases with increasing $M_{r}$, as shown in Table II. The values of $U(\mu, \sigma)$ and $V(\mu, \sigma)$ are also presented in this table, which will be used in evaluating user capacity via the iterative method, as described in Section III. The values of $\beta_{d}$ and $\beta_{v}$ are to be used in evaluating the multiclass CDMA system. Table III is similar to Table II, except that various numbers of transmit antenna elements are considered.

\section{A. User Capacity of CDMA}

Fig. 5 presents the reverse-link user capacity with beamforming and illustrates the effect of the number of receive antenna elements. Fig. 5(a) clearly shows that the user capacity increases significantly when the number of antenna elements increases. However, as shown in Fig. 5(b), the user capacity per antenna drops with the increase of antenna elements. In Fig. 5(a), we also compare the capacity evaluations using a simple closed-form expression (11) and those based on the complex iterative method, where the outage probability, as discussed in Section III, is assumed to be 0.05 [2]. Almost identical capacity results are obtained using the two evaluation methods. Based on numerical calculations, for some cases, they give identical results. In some other scenarios, they only differ by one user in capacity evaluations. For example, when $M_{r}=5$ and $M_{t}=1$, from (11) we obtain $N=144$. When the iterative method is used, we get $N=145$, which corresponds to an outage probability of 0.015 . However, a close examination reveals that the system with 145 users is not sustainable (not practical), since the mean other-cell interference
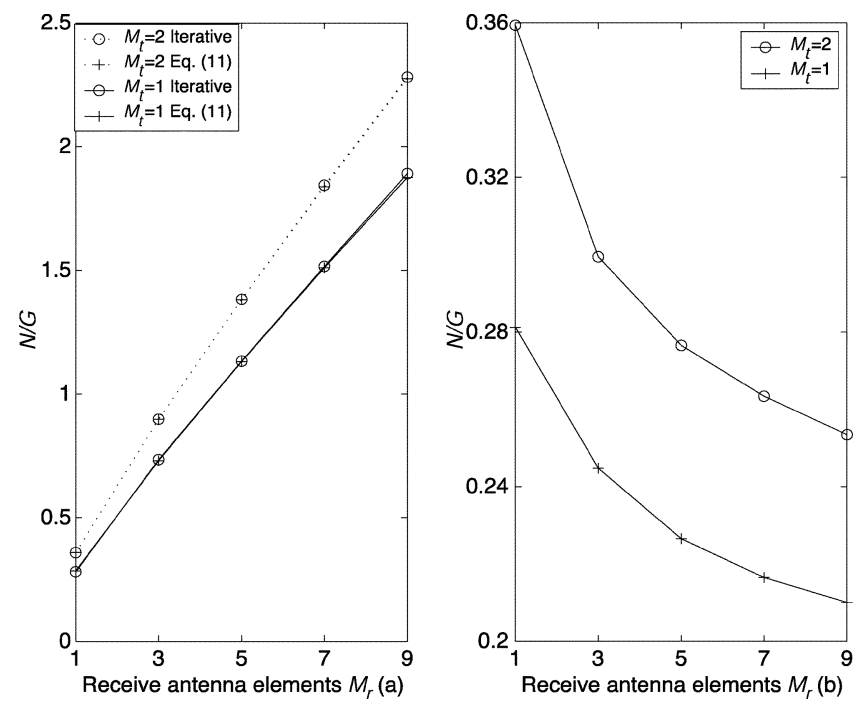

Fig. 5. User capacity. (a) Effect of beamforming and (b) per receive antenna element.

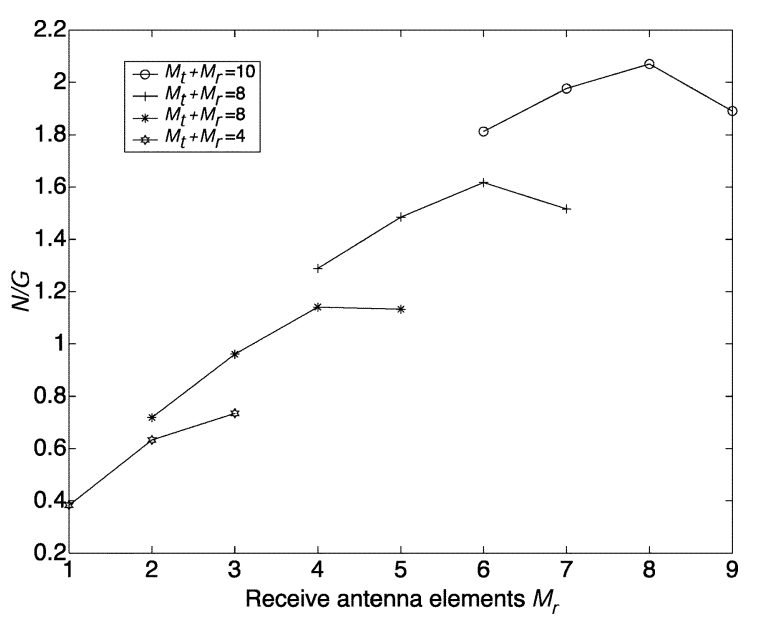

Fig. 6. User capacity, impact of antenna element distribution between the transmitter and receiver.

reaches $\mathrm{E}[I] \approx 1244 \eta_{0} W$. Note that, when $N=144$, we have $\mathrm{E}[I] \approx 114 \eta_{0} W$. Recall that the simple closed-form expression is derived in Section III to evaluate user capacity. Fig. 5(a) illustrates the effectiveness and accuracy of this approach.

Fig. 5 presents the capacity results of single-code CDMA systems, which are also applicable to multiclass CDMA systems when $g_{a}=1$ and let $N=N_{a} / g_{a}+N_{d} \beta_{d} / g_{d}+N_{v} \beta_{v} / g_{v}$, (11) and (21) are identical. This suggests that once $N$ is calculated, the values of $\left(N_{a}, N_{d}, N_{v}\right)$ can be determined by a BS according to $\left(g_{a}, g_{d}, g_{v}\right)$ and $\left(\beta_{d}, \beta_{v}\right)$.

For a fixed number of total antenna elements $\left(M_{t}+M_{r}\right)$, it is interesting to determine the distribution of antenna elements between the transmit and receive sides to maximize user capacity. Fig. 6 presents the capacity results when $M_{t}+M_{r}=4,6,8$, and 10. When $M_{t}+M_{r}=4$, it is seen that maximum capacity is achieved when $M_{t}=1,\left(M_{r}=3\right)$. For cases with $M_{t}+M_{r}=6,8$, and 10, maximum capacity is achieved when $M_{t}=2$. The numerical results indicate that, to obtain higher reverse-link user capacity, it is desirable to put more antenna elements at the receive sides (BS) compared to the transmit side 

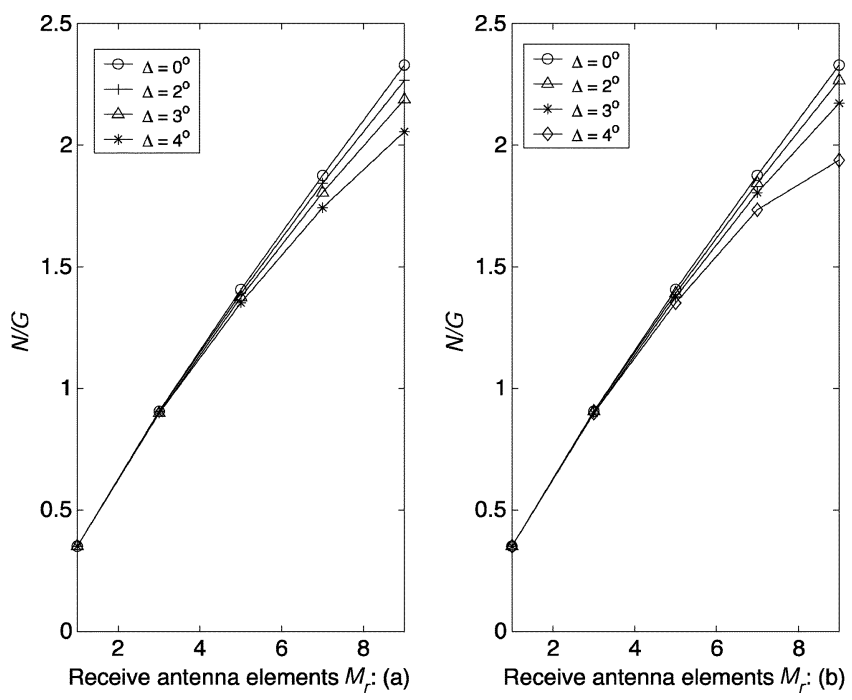

Fig. 7. User capacity, impact of DoA estimation errors. (a) Uniform distribution and (b) normal distribution.

(MS). The numerical results also suggest that when $M_{t}+M_{r}$ is small, say 4, only one antenna element should be placed at the transmit side. When $M_{t}+M_{r}$ increases to 6,8 , or 10 , two antenna elements should be used at the transmit side. With further increasing $M_{t}+M_{r}$, it is expected that the number of antenna elements at the transmit side should be increased.

\section{B. User Capacity Considering DoA Estimation Errors}

The capacity with beamforming considering DoA estimation errors can be evaluated using (13) and the corresponding numerical results of user capacity are shown in Fig. 7(a) and (b), where the number of transmit antenna elements $M_{t}$ is set to be two and that of receive antenna elements $M_{r}$ varies from 1 to 9. Both normal [Fig. 7(a)] and uniform [Fig. 7(b)] distribution of the estimated DoA errors are considered, in which $\Delta$ varies from $2^{\circ}$ to $4^{\circ}$. It is observed that the capacity reduction increases with increased estimation errors. Since the beamwidth becomes narrower with increased number of receive antenna elements, the same errors $(\Delta)$ can lead to a significant reduction of the capacity when $M_{r}$ is larger.

\section{User Capacity of CDMA With Beamforming and RAKE Receiver}

When both beamforming and a RAKE receiver are used, the reverse-link user capacity can be evaluated using (17). Assuming a multipart environment with $L=4$, the numerical results of user capacity are presented in Fig. 8(a) and (b). Both uniform [Fig. 8(a)] and exponential [Fig. 8(b)] PDPs are considered. For comparison, the number of transmit antenna elements $M_{t}$ is set to be 1 and 2 and the number of RAKE receiver fingers $R$ varies from 2, 3 to 4 . Noticeable capacity improvements are seen when the number of RAKE receiver fingers increases, which indicates the effectiveness of the RAKE receiver in combination with the use of beamforming.

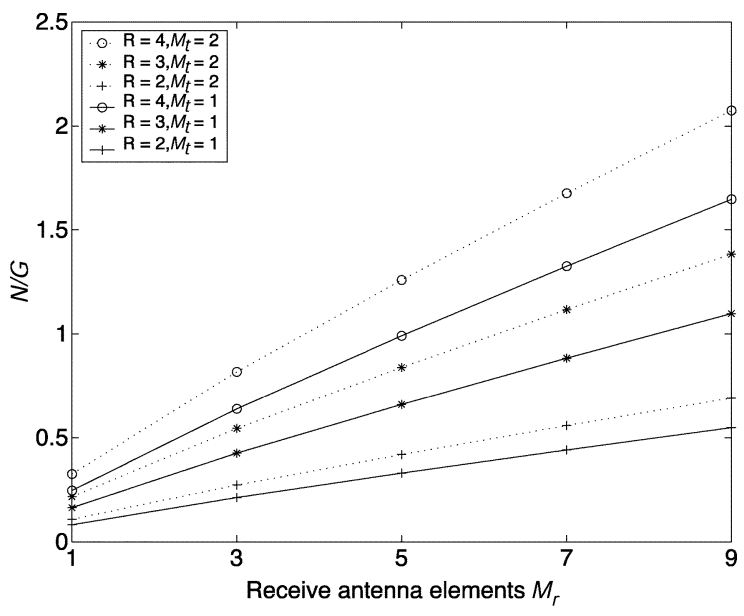

(a)

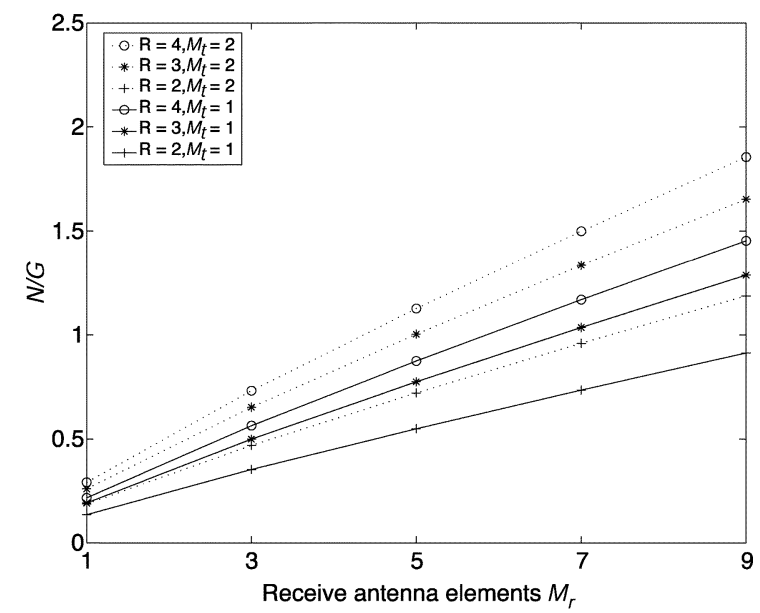

(b)

Fig. 8. User capacity, effect of beamforming and RAKE receiver. (a) Uniform PDP. (b) Exponential PDP.

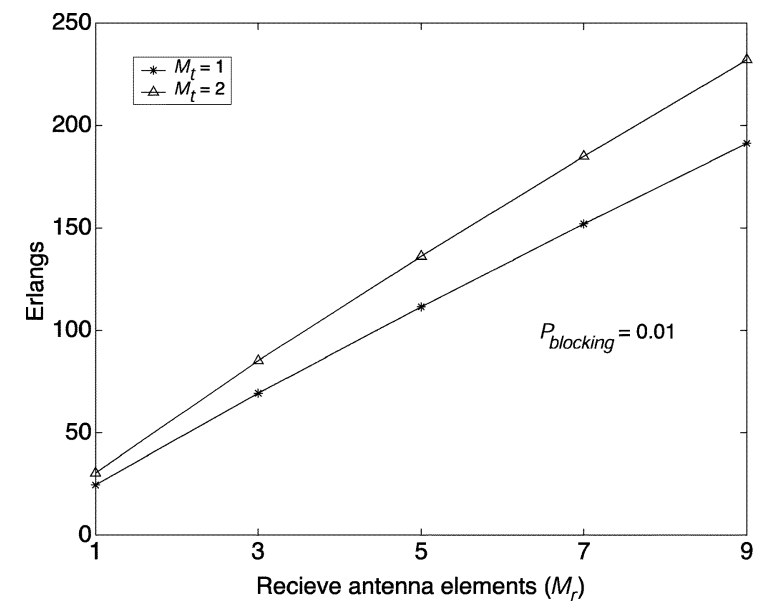

Fig. 9. Erlang capacity of a CDMA system with beamforming.

\section{Erlang Capacity of CDMA With Beamforming}

Erlang capacity due to different number of antenna elements at both transmit and receive sides are shown in Fig. 9. We consider $\gamma_{0}=5 \mathrm{~dB}$ and $P_{\text {blocking }}=0.01$. Following [14], the ratio of noise power to total noise-plus-interference power $\eta$ is assumed to be 0.1 . It is seen that Erlang capacity increases with 
an increase of the number of antenna elements at either side of the system. Given the Erlang capacity, we are able to calculate the number of users that a system can support under a specified call-blocking rate and traffic load. If the traffic load of each user is 0.1 , the system could support $84.62 / 0.1 \approx 846$ users dynamically with $M_{t}=2$ and $M_{r}=3$.

\section{CONCLUSION}

A simple closed-form expression is derived to evaluate reverse-link user capacity for CDMA and multiclass CDMA systems with beamforming and a RAKE receiver in multipath fading and multicell environments. The impact of the DoA estimation errors is also considered. The reverse-link Erlang capacity, which quantifies the dynamic traffic characterization, is analyzed. Both transmit and receive beamforming are assumed in the system and significant capacity improvements are observed with an increase in the number of antenna elements. The capacity improvement due to the RAKE receiver is also illustrated. The relationships between the capacity and various system parameters, including the target SNIR, CDMA processing gain, antenna array gain patterns, and the number of RAKE receiver fingers, are reflected in the simple closed-form capacity equations.

In this paper, we consider an LES array for beamforming. The developed capacity evaluation method can be applied to CDMA systems with other types of arrays by considering different transmit and receive array gain patterns $G_{t}$ and $G_{r}$. Perfect fast transmit power control is assumed in this paper. In a practical system, the loop delay of power control and the accuracy of SNIR estimation will have an impact on the performance of power control, which reduces the CDMA capacity. Furthermore, in a multipath environment, signals arrive at the receiver through multiple paths from different directions (angular spreads), which will increase the complexity to steer the beam toward each direction. All these practical issues affecting the system capacity will be studied in the future.

\section{APPENDIX A}

DERIVATION OF (11)

Equations (9) and (10) have to satisfy the following two conditions:

$$
\begin{aligned}
\mathrm{E}[I] & =\frac{1.5 N F(\mu, \sigma)}{C-N F(\mu, \sigma)} \eta_{0} W \geq 0 \\
\operatorname{Var}[I] & =\frac{(U(\mu, \sigma)-V(\mu, \sigma))}{\frac{C^{2}}{N}-U(\mu, \sigma)}\left(\mathrm{E}[I]+1.5 \eta_{0} W\right)^{2} \geq 0 .
\end{aligned}
$$

Using (28) and considering the fact that $F(\mu, \sigma)>0$, we get

$$
C-N F(\mu, \sigma)>0
$$

which gives

$$
N<\frac{\mathrm{G}_{\mathrm{r}}+\frac{1.5 G}{\gamma_{0}}}{\mathrm{G}_{\mathrm{r}}+F(\mu, \sigma)}
$$

And if $U(\mu, \sigma)>V(\mu, \sigma)$, from (29)

$$
C^{2}-U(\mu, \sigma) N>0
$$

Let $T=1.5 G / \gamma_{0}+\mathrm{G}_{\mathrm{r}}$ and substitute it into (31)

$$
\mathrm{G}_{\mathrm{r}}^{2} N^{2}+T^{2}-\left(2 T \mathrm{G}_{\mathrm{r}}+U(\mu, \sigma)\right) N>0 .
$$

Solving (32), we get

$$
N>\frac{2 T \mathrm{G}_{\mathrm{r}}+U(\mu, \sigma)+\sqrt{U(\mu, \sigma)+4 T \mathrm{G}_{\mathrm{r}} U(\mu, \sigma)}}{2 \mathrm{G}_{\mathrm{r}}^{2}}
$$

and

$$
N<\frac{2 T \mathrm{G}_{\mathrm{r}}+U(\mu, \sigma)-\sqrt{U(\mu, \sigma)+4 T \mathrm{G}_{\mathrm{r}} U(\mu, \sigma)}}{2 \mathrm{G}_{\mathrm{r}}^{2}} .
$$

It is easily shown that

$$
\frac{2 T \mathrm{G}_{\mathrm{r}}+U(\mu, \sigma)+\sqrt{U(\mu, \sigma)+4 T \mathrm{G}_{\mathrm{r}} U(\mu, \sigma)}}{2 \mathrm{G}_{\mathrm{r}}^{2}}>\frac{\mathrm{G}_{\mathrm{r}}+\frac{1.5 G}{\gamma_{0}}}{\mathrm{G}_{\mathrm{r}}+F(\mu, \sigma)} .
$$

Due to $U(\mu, \sigma) \ll 1, \mathrm{G}_{\mathrm{r}} \leq 1, T \gg U(\mu, \sigma) \mathrm{G}_{\mathrm{r}}$, and $T \gg$ $U(\mu, \sigma) / \mathrm{G}_{\mathrm{r}}$, we get

$$
\begin{gathered}
\left(\sqrt{T}-\sqrt{\frac{U(\mu, \sigma)}{\mathrm{G}_{\mathrm{r}}}}\right)\left(F(\mu, \sigma)+\mathrm{G}_{\mathrm{r}}\right)-\sqrt{T} \mathrm{G}_{\mathrm{r}} \\
=\sqrt{T} F(\mu, \sigma)-\sqrt{\frac{U(\mu, \sigma)}{\mathrm{G}_{\mathrm{r}}}}\left(F(\mu, \sigma)+\mathrm{G}_{\mathrm{r}}\right)>0 .
\end{gathered}
$$

Thus

$$
\frac{T-\sqrt{\frac{T U(\mu, \sigma)}{\mathrm{G}_{\mathrm{r}}}}}{\mathrm{G}_{\mathrm{r}}}>\frac{T}{F(\mu, \sigma)+\mathrm{G}_{\mathrm{r}}}=\frac{\mathrm{G}_{\mathrm{r}}+\frac{1.5 G}{\gamma_{0}}}{F(\mu, \sigma)+\mathrm{G}_{\mathrm{r}}} .
$$

Combining (30)-(36), $N$ has to satisfy

$$
N<\frac{\mathrm{G}_{\mathrm{r}}+\frac{1.5 G}{\gamma_{0}}}{F(\mu, \sigma)+\mathrm{G}_{\mathrm{r}}} .
$$

If $U(\mu, \sigma)<V(\mu, \sigma)$, from (30), (32), (33), and (34) we could conclude that there is no $N$ that satisfies (28) and (29) simultaneously.

\section{ACKNOWLEDGMENT}

The authors would like to thank the anonymous reviewers for their valuable comments.

\section{REFERENCES}

[1] J. E. Padgett, C. G. Gunther, and T. Hattori, "Overview of wireless personal communications," IEEE Commun. Mag., vol. 33, pp. 28-41, Jan. 1995. 
[2] D. K. Kim and F. Adachi, "Theoretical analysis of reverse link capacity of an SIR-based power-controlled cellular CDMA system in a multipath fading environment," IEEE Trans. Veh. Technol., vol. 50, pp. 452-464, Mar. 2001.

[3] M. Zeng, A. Annamalai, and V. K. Bhargava, "Recent advances in cellular wireless communications," IEEE Commun. Mag., vol. 36, pp. 128-138, Sept. 1999.

[4] K. S. Gilhousen et al., "On the capacity of a cellular CDMA system," IEEE Trans. Veh. Technol., vol. 40, pp. 303-312, May 1991.

[5] S. Verdu, Multiuser Detection. Cambridge, U.K.: Cambridge Univ. Press, 1998.

[6] D. N. C. Tse and S. V. Hanly, "Linear multiuser receivers: effective interference, effective bandwidth and user capacity," IEEE Trans. Inform. Theory, vol. 45, pp. 641-657, Mar. 1999.

[7] U. Madhow and M. Honig, "MMSE interference suppression for directsequence spread-spectrum CDMA," IEEE Trans. Commun., vol. 42, pp. 3178-3188, Dec. 1994.

[8] M. Xiao, N. B. Shroff, and E. K. P. Chong, "Resource management in power-controlled cellular wireless systems," Wireless Commun. Mob. Comput., vol. 1, no. 2, pp. 185-199, 2001.

[9] A. El-Osery and C. Abdallah, "Distributed power control in CDMA cellular systems," IEEE Antennas Propagat. Mag., vol. 42, Aug. 2000.

[10] A. J. Viterbi, A. M. Viterbi, and E. Zehavi, "Other-cell interference in cellular power-controlled CDMA," IEEE Trans. Commun., vol. 42, pp. 1501-2504, Feb./Mar./Apr. 1994

[11] D. K. Kim and D. K. Sung, "Capacity estimation for a multicode CDMA system with SIR-based power control," IEEE Trans. Veh. Technol., vol. 50, pp. 701-710, May 2001.

[12] — - "Capacity estimation for an SIR-based power-controlled CDMA system supporting on-off traffic," IEEE Trans. Veh. Technol., vol. 50, pp. 1094-1101, July 2000.

[13] S. J. Lee, H. W. Lee, and D. K. Sung, "Capacity of single-code and multicode DS-CDMA systems accommodating multiclass services," IEEE Trans. Veh. Technol., vol. 48, pp. 376-384, Mar. 1999.

[14] A. M. Viterbi and A. J. Viterbi, "Erlang capacity of a power controlled CDMA system," IEEE J. Select. Areas Commun., vol. 11, pp. 892-810, Aug. 1993.

[15] T. S. Rappaport, Wireless Communications: Principles and Practice, 2nd ed. Englewood Cliffs, NJ: Prentice-Hall, 2002

[16] J. B. Kim and M. L. Honig, "Resource allocation for multiple classes of DS-CDMA traffic," IEEE Trans. Veh. Technol., vol. 49, pp. 506-519, Mar. 2000.

[17] J. C. Liberti and T. S. Rappaport, Smart Antennas for Wireless Communications: IS-95 and Third Generation CDMA Applications. Englewood Cliffs, NJ: Prentice-Hall, 1999.

[18] F. Rashid-Farrokhi, K. J. R. Liu, and L. Tassiulas, "Transmit beamforming and power control for cellular wireless systems," IEEE J. Select. Areas Commun., vol. 16, pp. 1437-1450, Oct. 1998.

[19] S. Haykin, Adaptive Filter Theory, 3rd ed. Englewood Cliffs, NJ: Prentice-Hall, 1986

[20] H. L. Van Trees, Optimum Array Processing, Part IV of Detection, Estimation, and Modulation Theory. New York: Wiley, 2002.

[21] P. Pajusco, "Experimental characterization of DOA at the base station in rural and urban area," in Proc. IEEE Vehicular Technology Conf., May 1998, pp. $18-21$.

[22] M. Toeltsch et al., "Statistical characterization of urban spatial radio channels," IEEE J. Select. Areas. Commun., vol. 20, pp. 539-549, Apr. 2002 .

[23] J. Chen, J. Wang, and M. Sawahashi, "MCI cancellation for multicode wideband CDMA systems," IEEE J. Select. Areas. Commun., vol. 20, pp. 450-462, Feb. 2002.

[24] Z. Liu, M. J. Karol, M. E. Zarki, and K. Y. Eng, "A demand-assignment access control for multicode DS-CDMA wireless packet (ATM) networks," in Proc. IEEE INFOCOM'96, San Francisco, CA, May 1996, pp. 713-721.

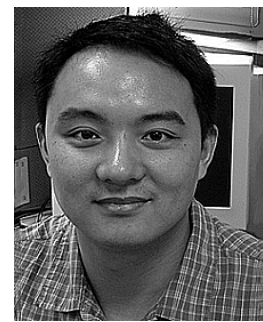

Jin Yu (S'01) received the B.S.E.E. degree from Wuhan University, China, in 1998, the M.S.E.E. degree from the University of Mississippi, Oxford, in 2001, and is currently working toward the Ph.D degree in electrical engineering at Stevens Institute of Technology, Hoboken, NJ.

During his study at the University of Mississippi, his research work focused on the computational electromagnetics and microwave circuits. At present, he is doing research in the applications of phased arrays in wireless communications at the Wireless Information Systems Engineering Laboratory (WISELAB), Stevens Institute of Technology. His research interest areas include code-division multiple access (CDMA), signal processing for wireless communications, and adaptive antennas.

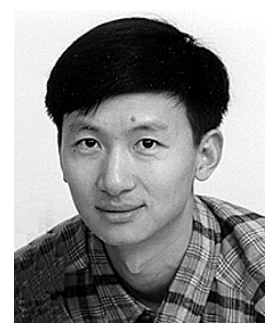

Yu-Dong Yao (S'88-M'88-SM'94) received the B.Eng. and M.Eng. degrees from Nanjing University of Posts and Telecommunications, Nanjing, China, in 1982 and 1985, respectively, and the Ph.D. degree from Southeast University, Nanjing, China, in 1988, all in electrical engineering.

From 1989 to 1990, he was with Carleton University, Ottawa, ON, Canada, as a Research Associate working on mobile radio communications. From 1990 to 1994, he was with Spar Aerospace Ltd. Montreal, PQ, Canada, where he was involved in research on satellite communications. From 1994 to 2000, he was with Qualcomm Inc., San Diego, CA, where he participated in research and development in wireless code-division multiple-access (CDMA) systems. He joined the Stevens Institute of Technology, Hoboken, NJ, in 2000, where he is an Associate Professor in the Department of Electrical and Computer Engineering and a Director of the Wireless Information Systems Engineering Laboratory (WISELAB). He holds one Chinese patent and eight U.S. patents. He was a Guest Editor for a Special Issue on wireless networks for the International Journal of Communication Systems. His research interests include wireless communications and networks, spread spectrum and CDMA, and DSP for wireless systems.

Dr. Yao is an Associate Editor of IEEE COMMUNICATIONS LETTERS and IEEE TRANSACTIONS ON VehiculaR TECHNOLOGY and an Editor for IEEE TRANSACTIONS ON WIRELESS COMMUNICATIONS.

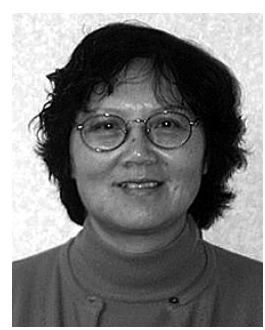

Jinyun Zhang received the Ph.D. degree in electrical engineering from the University of Ottawa, Ottawa, ON, Canada, in 1991

She was with Nortel Networks for more than 10 years, where she held engineering and management positions in the areas of very-large-scale integration (VLSI) design, advanced wireless technology development, and wireless and optical networks. She currently is a Senior Principal Technical Staff Member and Group Manager of the digital communication and networking group with Mitsubishi Electric Research Laboratories (MERL), Cambridge, MA, where she manages many wireless communications and networking projects, including UWB, IEEE802.11WLAN, ZigBee, sensor network, and 3G/4G wireless communications. 